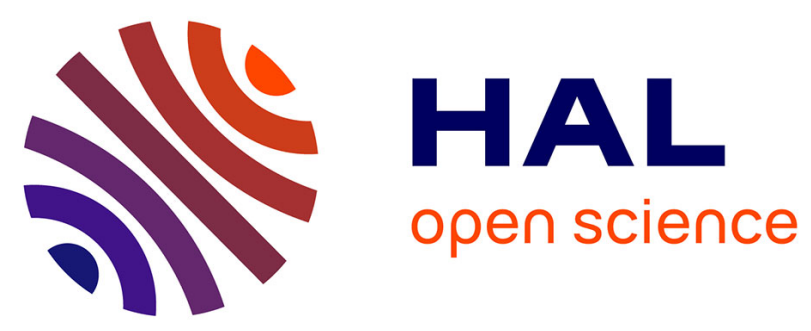

\title{
Vortical flow calculations using a high-order Vorticity Confinement method
}

\author{
Ilias Petropoulos, Michel Costes, Paola Cinnella
}

\section{To cite this version:}

Ilias Petropoulos, Michel Costes, Paola Cinnella. Vortical flow calculations using a high-order Vorticity Confinement method. 23rd AIAA Computational Fluid Dynamics Conference, Jun 2017, Denver, United States. 10.2514/6.2017-3291 . hal-03449105

\section{HAL Id: hal-03449105 \\ https://hal.science/hal-03449105}

Submitted on 25 Nov 2021

HAL is a multi-disciplinary open access archive for the deposit and dissemination of scientific research documents, whether they are published or not. The documents may come from teaching and research institutions in France or abroad, or from public or private research centers.
L'archive ouverte pluridisciplinaire HAL, est destinée au dépôt et à la diffusion de documents scientifiques de niveau recherche, publiés ou non, émanant des établissements d'enseignement et de recherche français ou étrangers, des laboratoires publics ou privés. 


\title{
Vortical flow calculations using a high-order Vorticity Confinement method
}

\author{
Ilias Petropoulos* and Michel Costes ${ }^{\dagger}$ \\ ONERA, The French Aerospace Lab, 8 rue des Vertugadins, F-92190 Meudon, France \\ Paola Cinnella \\ DynFluid Laboratory, Arts et Métiers ParisTech, 151 Blvd de l'Hôpital, F-75013 Paris, France
}

\begin{abstract}
A high-order Vorticity Confinement (VC) method is applied to the calculation of compressible vortical flows. High-order extensions of the VC methodology have been developed for the Navier-Stokes equations using a methodology that remains independent of the baseline numerical scheme, as the original VC formulation of Steinhoff. The VC method is primarily oriented towards the accurate calculation of cases involving the advection of vortical structures (e.g. wakes), allowing their propagation over long distances with low numerical dissipation. The present work however mainly investigates their applicability to the simulation of turbulent flows, where the improvement in the preservation of vortical structures shows great interest. High-order VC schemes were found to be consistent with complex vortical flow dynamics without the need of special treatment to accommodate the vortex interaction mechanisms characteristic of turbulent flows. Furthermore, they were found to consistently improve the resolvability of baseline FE-MUSCL schemes for reasonable values of the confinement parameter $\varepsilon$, close to the value of the high-order artificial dissipation coefficient. Last, the dependence of the solution on the confinement parameter $\mu$ was found to be moderate for the values and cases investigated in this paper.
\end{abstract}

\section{Introduction}

The understanding and accurate prediction of vortical flows is a subject of great interest in a wide range of modern industrial and research applications. Experimental methods have shown remarkable progress over the past years, ${ }^{1,2}$ with the emergence of techniques being capable of detecting complex flow phenomena even in realistic configurations. They are still however costly and time-demanding, meaning that broad explorative studies using experimental methods will continue to be unattainable in the near future.

Computational fluid dynamics methods have been a primary focus of modern research, due to their broad applicability in engineering applications. Despite the significant evolution and overall competency of numerical methods, the calculation of vortical flows remains a challenge. Numerical schemes must be dissipative to ensure the damping of spurious oscillations, which in turn causes the spreading and diffusion of vortical structures in simulations. Lagrangian solvers allow the propagation of vortical structures with minimal dissipation,,$^{3-5}$ but their application remains limited to research for advanced vortical flow simulations. On the other hand, the more robust formulation of Eulerian methods has lead to their wide adoption by both research and industry over the past decades.

Hybrid structured-unstructured Eulerian ${ }^{6,7}$ or Eulerian-Lagrangian solvers ${ }^{8}$ can allow the calculation of each flow region by the most efficient approach. In pure structured or unstructured solvers, the problem of spurious vortex dissipation is usually treated by the use of finer meshes or automatic mesh adaptation methods,,${ }^{9} 10$ which often requires a considerable increase of computational cost and algorithmic complexity. A more fundamental approach consists in the use of high-order methods, which provide a more precise approximation of the fluid dynamics equations. In the widely adopted Finite Volume formulation, the

*PhD Student, ONERA Department of Aerodynamics, Aeroelasticity and Acoustics, ilias.petropoulos@onera.fr.

${ }^{\dagger}$ Research Scientist, ONERA Department of Aerodynamics, Aeroelasticity and Acoustics, michel.costes@onera.fr.

¥Professor, DynFluid Laboratory, Arts et Métiers ParisTech, paola.cinnella@ensam.eu. 
implementation of truly high-order flux derivatives is complicated, especially for curvilinear and unstructured grids $^{11}$ whereas the dissipation of vortices still cannot be completely prevented.

Another alternative consists in the development of numerical techniques that are specifically oriented towards the accurate calculation of vortical flows. These can be solvers of the vorticity-velocity formulation of the Navier-Stokes equations ${ }^{12,13}$ or numerical schemes ensuring that no dissipation term is introduced in the discrete vorticity transport equation. ${ }^{14,15}$ Another technique is the Vorticity Confinement (VC) method of Steinhoff ${ }^{16-18}$ designed to capture small-scale features of high-Reynolds number vortical flows by introducing a negative dissipation term in the flow equations to balance the excess dissipation in vortical regions. This method has been widely applied in the aeronautics field, its important advantage being that it is formulated independently of the baseline numerical scheme.

The original method is however $1^{\text {st }}$-order accurate, meaning that vortex profiles are rapidly governed by the VC term in high-order simulations and the order of accuracy deteriorates in vortical regions. ${ }^{19}$ Due to the growth of high-order methods, the construction of a Vorticity Confinement method that is appropriate for high-order computations shows great interest. High-order extensions of the method were developed for the linear transport equation ${ }^{20}$ and results showed that the numerical scheme's asymptotic solution is the same at all orders of accuracy, but the rate of convergence is slower at higher orders. On this basis, high-order extensions of the conservative VC2 formulation of Steinhoff have been developed for the Euler and Navier-Stokes equations based on the Laplacian of the original VC term. ${ }^{19,21}$ These extensions remain independent of the baseline numerical scheme and rotationally invariant, as the original method of Steinhoff. Furthermore, results show that they maintain the vorticity-preserving efficiency of the original method and are appropriate for high-order simulations as they preserve the global high-order of accuracy in vortical regions.

The Vorticity Confinement method has been shown to be capable of improving the preservation of vorticity in simulations, even in cases with significant changes in vortex formation and topology. ${ }^{22}$ However, aerodynamic applications of the method to the simulation of turbulent flows remain limited, ${ }^{18,23}$ and mostly concern the $\mathrm{VC} 1$ formulation. The objective of the present work is the evaluation of high-order extensions of the VC2 method and their applicability to the simulation of compressible turbulent flows. Section II presents high-order Vorticity Confinement schemes for the scalar transport and Navier-Stokes equations, and reviews their spectral properties compared to baseline upwind FE-MUSCL schemes. Section III presents numerical results from the application of high-order VC to the simulation of compressible vortical flows (vortex advection, Taylor-Green vortex, homogeneous isotropic turbulence decay) and conclusions are discussed in Section IV.

\section{Description of the schemes}

\section{II.A. Linear transport equation}

Confinement schemes for the linear transport equation were previously developed and analyzed for high-order extensions of the Lax-Wendroff and Beam-Warming schemes. ${ }^{20}$ This section presents a scalar high-order formulation of confinement for upwind non-compact flux discretizations ${ }^{21}$ which are decoupled in space and time. In the scalar case of the linear transport equation

$$
u_{t}+a u_{x}=0 \quad, a>0
$$

the VC method will be simply referred to as "confinement" since the transported variable does not specifically correspond to vorticity. The semi-discrete approximation of Eq. (1) can be written in the generic conservative form:

$$
\frac{\partial u}{\partial t}+\frac{a}{h_{j}}\left(F_{j+\frac{1}{2}}-F_{j-\frac{1}{2}}\right)=0
$$

where $h$ is the mesh spacing and $F_{j+\frac{1}{2}}$ is the numerical flux of the space discretization scheme on the interface

$x_{j+\frac{1}{2}}$. For uniform mesh spacing $h\left(x_{j}=j h, j \in \mathbb{Z}\right)$, the spatial derivative of Eq. (1) can be approximated at $10^{t h}$-order of accuracy using a linear non-compact centered discretization: ${ }^{24,25}$

$$
\frac{\partial(\bullet)}{\partial x}=\frac{1}{h} \delta \mu\left(I-\frac{1}{6} \delta^{2}+\frac{1}{30} \delta^{4}-\frac{1}{140} \delta^{6}+\frac{1}{630} \delta^{8}\right)(\bullet)+\mathcal{O}\left(h^{10}\right)
$$


where $\delta(\bullet)_{j+\frac{1}{2}} \triangleq(\bullet)_{j+1}-(\bullet)_{j}$ and $\mu(\bullet)_{j+\frac{1}{2}} \triangleq \frac{1}{2}\left((\bullet)_{j+1}+(\bullet)_{j}\right)$ are respectively the difference and average operators over a grid cell. Eq. (3) can be obtained by recursively correcting the leading truncation error term of a $2^{\text {nd }}$-order discretization. By recursively suppressing the $\delta^{8}-, \delta^{6}-, \delta^{4}$ - and $\delta^{2}$ terms, the resulting approximation is $8^{\text {th }}, 6^{\text {th }}, 4^{\text {th }}$ and $2^{\text {nd }}$-order accurate respectively. Since the centered approximation of the spatial derivative is non-dissipative, a linear high-order dissipation term is added to ensure the damping of spurious oscillations. The numerical flux of the space discretization at $p^{\text {th }}$ (odd)-order of accuracy is then written:

$$
F_{j+\frac{1}{2}}^{p}=\mu\left(\sum_{l=0}^{(p-1) / 2} b_{l} \delta^{2 l}\right) u_{j}-k_{p} \delta\left(\delta^{p-1}\right) u_{j}
$$

where the first term corresponds to the centered convective flux discretization and the second to artificial dissipation. Above, $b_{l}$ are the real coefficients in Eq. (3) and $k_{p}$ is a real constant called the artificial dissipation coefficient. For $k_{p}=\frac{1}{2} b_{\frac{p-1}{2}}$ the dissipation of the scheme is equivalent to that of a non-compact upwind scheme. ${ }^{25}$ The numerical flux of Eq. (4) is then equivalent to non-limited MUSCL schemes based on flux extrapolation (FE-MUSCL).

The idea of confinement is to introduce an additional nonlinear negative dissipation term in the flux discretization of Eq. (4), to balance the excess dissipation of the baseline numerical scheme. This confinement term is built on the same operator as the scheme's high-order artificial dissipation, but using the harmonic mean:

$$
(\tilde{h}(\bullet))_{j}=\left\{\begin{array}{lll}
\frac{2(\bullet)_{j}(\bullet)_{j-1}}{(\bullet)_{j}+(\bullet)_{j-1}} & \text {, if } \quad(\bullet)_{j}(\bullet)_{j-1}>0 \\
0 & \text {,if } \quad(\bullet)_{j}(\bullet)_{j-1} \leq 0
\end{array}\right.
$$

of the transported variable at each point of the stencil, instead of the transported variable itself. With this choice of dissipation operator the nonlinear confinement term introduces negative dissipation according to the dissipative error of the baseline scheme. The numerical flux of the scheme with confinement at $p^{\text {th }}$ (odd)-order of accuracy then writes:

$$
F_{j+\frac{1}{2}}^{p}=\mu\left(\sum_{l=0}^{(p-1) / 2} b_{l} \delta^{2 l}\right) u_{j}-k_{p} \delta\left(\delta^{p-1}\right) u_{j}+\varepsilon \delta\left(\delta^{p-1}\right)(\tilde{h}(u))_{j}
$$

where $\varepsilon$ is a real constant called the confinement parameter, usually chosen in the order of the artificial dissipation coefficient. For the flux discretization of Eq. (6), the scheme will be referred to as the $p^{t h}$-order FE-MUSCL with confinement.

\section{II.B. Navier-Stokes equations}

The present section presents high-order extensions of the VC2 Vorticity-Confinement formulation of Steinhoff ${ }^{26,27}$ for the compressible Euler and Navier-Stokes equations. The VC method consists in introducing a rotational vorticity-based negative dissipation term in the Navier-Stokes momentum equation as a source term, which balances the baseline numerical dissipation in vortical flow regions. For the original VC2 formulation of Steinhoff the conservative differential form of the momentum conservation equation is written:

$$
\frac{\partial \rho \vec{v}}{\partial t}+\vec{\nabla} \cdot(\rho \vec{v} \otimes \vec{v}+p \overline{\bar{I}}-\overline{\bar{\tau}})=\vec{f}
$$

with the Vorticity Confinement term:

$$
\vec{f}=-\vec{\nabla} \times(\mu \vec{\omega}-\varepsilon \overrightarrow{\mathrm{w}})
$$

The first part is a linear artificial dissipation term with coefficient $\mu$ aligned with the vorticity vector $\vec{\omega}=\vec{\nabla} \times \vec{v}$. The second part is the negative dissipation confinement term with coefficient $\varepsilon$ multiplied with the vector $\overrightarrow{\mathrm{w}}$ :

$$
\overrightarrow{\mathrm{w}}=\frac{\vec{\omega}}{\|\vec{\omega}\|} \tilde{h}\left(\omega_{j}\right)=\frac{\vec{\omega}}{\|\vec{\omega}\|} N\left(\sum_{j=1}^{N}\left\|\vec{\omega}_{j}\right\|^{-1}\right)^{-1}
$$

which is aligned with vorticity and has a magnitude equal to the harmonic mean of the modulus of vorticity around the neighboring cells. 
The VC term balances the excess dissipation in vortical regions through the second term of Eq. (8), which represents a negative nonlinear dissipation in analogy with the scalar formulation in Eq. (6). In principle, the positive dissipation term is not necessary and its introduction may appear counterintuitive. For the scalar case presented in Section II.A, its role is taken by the high-order dissipation of the baseline scheme. For the Navier-Stokes equations however, the first term of Eq. (8) is different from the baseline dissipation since it is explicitly based on vorticity and independent of the baseline numerical method. The consistency of the discretization of Eq. (7) is ensured by using undivided differences in the computation of the curl of the VC term.

High-order extensions can be obtained by recursively applying the curl operator on the VC term of Eq. (8) to increase the order of differencing, in analogy with the $\delta$ operator in the linear case. ${ }^{19,21}$ The alternate sign of high-order derivatives is then naturally introduced. A $3^{\text {rd }}$-order $\mathrm{VC}$ term can be obtained by applying twice the curl operator on $\vec{f}$ as:

$$
\begin{aligned}
\vec{\nabla} \times \vec{f} & =-\vec{\nabla} \times \vec{\nabla} \times(\mu \vec{\omega}-\varepsilon \overrightarrow{\mathrm{w}})=-\vec{\nabla}(\vec{\nabla} \cdot(\mu \vec{\omega}-\varepsilon \overrightarrow{\mathrm{w}}))+\vec{\nabla}^{2}(\mu \vec{\omega}-\varepsilon \overrightarrow{\mathrm{w}}) \\
\overrightarrow{f_{3}} & =\vec{\nabla} \times \vec{\nabla} \times \vec{f}=\vec{\nabla} \times\left(\vec{\nabla}^{2}(\mu \vec{\omega}-\varepsilon \overrightarrow{\mathrm{w}})\right)
\end{aligned}
$$

The specific vorticity transport equation can be derived by taking the curl of Eq. (7) and dividing by density. For an isolated two-dimensional vortex in inviscid flow and the $3^{\text {rd }}$ order VC term of Eq. (10) this is written:

$$
\frac{\partial(\vec{\omega} / \rho)}{\partial t}+\vec{v} \cdot \vec{\nabla}(\vec{\omega} / \rho)-\frac{1}{\rho} \vec{\nabla}^{2}\left(\vec{\nabla}^{2}(\mu \vec{\omega}-\varepsilon \overrightarrow{\mathrm{w}})\right)=0
$$

Above, the last term corresponds to the contribution of the $3^{r d}$-order VC term of Eq. (10) and is equal to the Laplacian of the contribution of the original $1^{\text {st }}$-order term, indicating similar dynamics from the balance of the positive and negative dissipation parts of the $\mathrm{VC}$ term. ${ }^{19}$

Equivalently, a $5^{t h}$-order VC term can be obtained by applying twice the curl operator on $\vec{f}_{3}$ as:

$$
\overrightarrow{f_{5}}=\vec{\nabla} \times \vec{\nabla} \times \overrightarrow{f_{3}}=-\vec{\nabla} \times\left(\vec{\nabla}^{4}(\mu \vec{\omega}-\varepsilon \overrightarrow{\mathrm{w}})\right)
$$

The use of undivided differences in the computation of the Laplacian and bi-Laplacian operator in Eqs. (10), (12) ensures consistency with Eq. (7) and provides the increased order of accuracy. High-order VC extensions based on the Laplace operator remain both independent of the baseline numerical method and rotationally invariant, in consistency with the original VC formulation of Steinhoff. The VC2 formulation of Eq. (8) is conservative, a property which can be made apparent by deriving the integral form of the momentum equation (7) and assimilating the VC term alongside physical fluxes as: ${ }^{21}$

$$
\frac{\partial}{\partial t} \int_{\Omega} \rho \vec{v} d \Omega+\oint_{S}[(\rho \vec{v} \otimes \vec{v}+p \overline{\bar{I}}-\overline{\bar{\tau}}) \cdot \vec{n}-(\mu \vec{\omega}-\varepsilon \overrightarrow{\mathrm{w}}) \times \vec{n}] d S=0
$$

showing that the $\mathrm{VC} 2$ term is equivalent to a nonlinear anti-diffusive flux correction applied in vortical regions. The same stands for the high-order VC2 extensions of Eqs. (10),(12). It should be noted that these schemes are not similar to high-order extensions of linear flux discretizations, which aim at a more precise approximation of inviscid fluxes, but represent a nonlinear high-order flux correction explicitly based on vorticity that can be applied independently of the baseline numerical scheme.

The confinement parameters $\mu, \varepsilon$ are constant in space and time and have the dimensions of density times velocity. For computations involving dimensional quantities in the present work, their values were dimensionalized as $\varepsilon^{*}=\rho_{\text {ref }} V_{\text {ref }} \varepsilon$. Throughout the presentation of numerical results in Section III their values are expressed as $\varepsilon, \mu / \varepsilon$, as the first defines the magnitude of the $\mathrm{VC}$ flux correction and the second represents the ratio between the positive and negative dissipation within the $\mathrm{VC}$ term. The $\mathrm{VC}$ method is applied to correct the excess dissipation of the baseline scheme in vortical regions and therefore the value of the coefficient $\mu$, which represents an additional dissipation, should be kept as small as possible. It could however be increased to introduce some stabilizing rotational dissipation in cases where the dissipation of the baseline scheme is not enough to balance the nonlinear negative dissipation of VC. For numerical schemes with explicitly known artificial dissipation the value of $\varepsilon$ is taken in the order of the artificial dissipation coefficient, in analogy with the scalar confinement formulation of Section II.A.

The identification of vortical regions to apply $\mathrm{VC}$ is performed by defining a minimum cut-off value of the Q-criterion. ${ }^{28}$ It is however practically used to avoid the introduction of spurious vorticity concentration 
by the introduction of negative dissipation in irrotational flow regions, rather than to precisely select the structures to apply the method. The cut-off value was set to 0.10 for all cases presented in this paper. Second, in analogy with the harmonic mean definition of Eq. (5) and since the VC correction should be applied in clearly defined vortical structures, $\mathrm{VC}$ is not applied if vorticity changes sign between the neighboring cells.

Presented calculations were performed with the DynHoLab Finite Volume solver of the DynFluid Laboratory. ${ }^{29}$ Physical fluxes are separated in an inviscid and viscous part, and discretized separately for each direction. Taking $W=(\rho, \rho \vec{v}, \rho E)^{T}$ the state vector of conservative variables and $f_{d}=f_{d}(W)$ the inviscid flux vector in the $d^{\text {th }}$ space direction, inviscid flux derivatives are approximated using non-compact centered approximations ${ }^{24,25}$ and the damping of spurious oscillations is achieved by introducing a high-order dissipation term. The numerical flux of the baseline discretization at $p^{t h}$-order of accuracy is:

$$
\mathcal{F}_{j+\frac{1}{2}}^{d}=\mu_{d}\left(\sum_{l=0}^{(p-1) / 2} b_{l} \delta_{d}^{2 l}\right) f_{d}-\frac{1}{2} b_{\frac{p-1}{2}}\left|Q_{d}\right| \delta_{d}^{p} W
$$

where $\delta_{d}$ and $\mu_{d}$ are the difference and average operators and $\left|Q_{d}\right|$ is the Roe linearization of the Jacobian matrix in direction $d$. The inviscid flux discretizations of Eq. (14) are equivalent to high-order upwind MUSCL schemes based on flux extrapolation and will be referred to as FE-MUSCL schemes, in analogy with the flux discretizations of Eq. (4) for the scalar transport equation. Viscous fluxes are approximated on cell face centers using a standard 3-point compact formula that is $2^{\text {nd }}$-order accurate on regular Cartesian grids. For the periodic flows presented, layers of ghost cells are introduced to expand the computational domain so that the same large-stencil schemes can be used for the calculation of fluxes on domain or zone boundaries.

The Vorticity Confinement term is added to the right-hand side of the Navier-Stokes momentum equation as a source term. For the high-order extensions of Eqs. (10), (12) the Laplace operator is computed on a 5 -point scheme, which was found to be very robust and more computationally efficient than a 3-point scheme similar to the one used for the calculation of viscous fluxes. The numerical calculation of the vector Laplacian of high-order VC is greatly simplified on a Cartesian coordinate system, as cross derivatives cancel and it degrades to the scalar Laplacian of each vector component. Finally, the computation of derivatives for the $\mathrm{VC}$ term is performed successively in the extended computational domain so that the VC term is accurately computed on the domain boundary. To ensure consistency with high-order flux discretizations ${ }^{19,21}$ the order of $\mathrm{VC}$ is always taken equal to the order of the baseline scheme for all cases presented in Section III.

\section{II.C. Spectral properties}

The dispersive and dissipative properties of the intrinsically nonlinear confinement schemes are presented for the scalar formulation of confinement presented in Subsection II.A. A classical linear spectral analysis can be performed by considering a linearization of the harmonic mean of Eq. (5) on a uniform grid using exponent functions. Such an approach however yields simplified results that do not match observations from the application of the schemes in numerical experiments. ${ }^{19,21,30}$

A more detailed representation can be obtained by evaluating the numerical schemes' spectral properties via application to short-time advection of a series of monochromatic sinusoidal initial conditions for the 1D linear transport equation (Eq. (1)). For sufficiently small time the computed solution remains monochromatic and the time integration error diminishes. The modified wavenumber $\xi^{*}$ of the space discretization scheme can be subsequently retrieved from the complex amplitude of the initial and computed solution. This procedure, originally applied by Pirozzoli for the study of shock-capturing schemes, ${ }^{31}$ is consistent with analytical results for linear schemes and provides an improved prediction of non-linear mechanisms.

Results of the analysis are shown in Fig. 1 for FE-MUSCL schemes with and without confinement up to $7^{\text {th }}$-order of accuracy. Naturally, higher-order schemes provide an accurate approximation of the exact solution for a longer range of wavenumbers. Results are presented for $\varepsilon=1.14 k_{p}$, a value that has commonly been applied in 1D calculations. Values in the same order of magnitude however produce similar trends, whereas the sensitivity to the value of $\varepsilon$ is smaller at higher orders. Schemes with confinement are shown to be stable $\left(\operatorname{Im}\left(\xi^{*}\right) \leq 0\right)$ and have improved spectral properties compared to baseline FE-MUSCL schemes, both in terms of dispersion and dissipation. The confinement term in Eq. (6) is originally of dissipative nature, the improvement of dispersive properties being a result of its nonlinear mechanism. An exception is observed for a discrete set of wavenumbers $k$ (i.e. reduced wavenumbers $\xi=k h$ ), for which the initial condition is such that $(\tilde{h}(u))_{j}=0$ for every position on the computational grid. In those cases the effect 
of the confinement term diminishes and the spectral properties match those of the baseline scheme. This weakness of confinement however refers to single harmonics and was not observed in general problems, whereas frequencies higher than $\pi / 2$ are under-resolved on the computational grid and should in any case be damped to avoid the generation of spurious oscillations. Finally, the nonlinear negative dissipation of confinement diminishes gradually at higher wavenumbers and does not modify the damping of spurious $2 \Delta$ waves. For a comprehensive discussion of the spectral properties of these schemes the reader is referred to Ref. $^{21}$
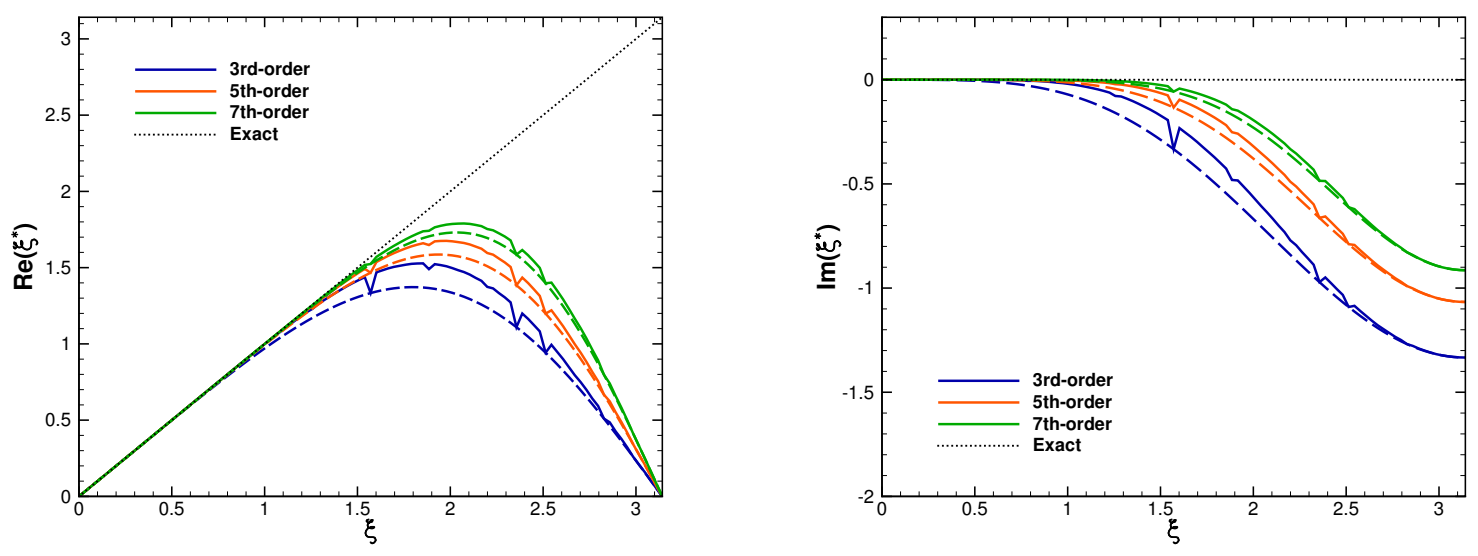

Figure 1. Spectral properties of FE-MUSCL schemes up to $7^{t h}$-order of accuracy. Baseline schemes (dashed lines) and with confinement (solid lines), $\varepsilon=1.14 k_{p}$. Taken from Ref. ${ }^{21}$

The resolvability limit of the schemes can be calculated by identifying the maximum reduced wavenumber $\xi$ up to which the spectral properties of the scheme approximate the exact solution under an error $E \leq 10^{-3}$. This limit is presented in Table 1 in the form of minimum number of points-per-wavelength $\lambda_{n} / h$. The leading error term of FE-MUSCL schemes is dissipative therefore their accuracy limit is primarily defined by dissipation, rather than dispersion. Confinement is shown to introduce a considerable reduction of this limit at all orders of accuracy, both in terms of dispersion and dissipation. The minimum number of pointsper-wavelength is halved at $3^{r d}$-order, while even at $5^{\text {th }}$ and $7^{\text {th }}$-order it tends towards the $1 \mathrm{D}$ resolvability limit of compact schemes of the same order of accuracy. Last, the improvement introduced by confinement is smaller at higher orders, in line with the reduced numerical error of the high-order baseline schemes.

Table 1. Resolvability limit in terms of number of points-per-wavelength $\lambda_{n} / h$ for FE-MUSCL schemes with and without confinement $\left(\varepsilon=1.14 k_{p}\right)$

\begin{tabular}{cccccc}
\hline \hline & \multicolumn{2}{c}{ Limit due to dispersion } & & \multicolumn{2}{c}{ Limit due to dissipation } \\
\cline { 2 - 3 } \cline { 5 - 6 } Scheme order & baseline & confinement & & baseline & confinement \\
& $\lambda_{n} / h$ & $\lambda_{n} / h$ & & $\lambda_{n} / h$ & $\lambda_{n} / h$ \\
\hline $3^{\text {rd }}$ & 13.33 & 6.45 & & 20.00 & 9.52 \\
$5^{\text {th }}$ & 8.33 & 6.25 & & 10.00 & 7.41 \\
$7^{\text {th }}$ & 6.45 & 5.56 & & 7.14 & 6.06 \\
\hline \hline
\end{tabular}

\section{Numerical results}

\section{III.A. Vortex advection}

The use of directional schemes in the discretization of fluid dynamics equations leads to grid directions being privileged in terms of numerical error, greatly influencing the accuracy of computations involving the advection of vortices. Computational meshes for simple applications can be appropriately constructed to follow the propagation direction of primary structures, but the same cannot be done for the majority of 
turbulent flows, which are naturally irregular. It is therefore necessary that numerical schemes are pertinently evaluated for such scenarios.

The present case consists in the long-distance advection of a 2-dimensional vortex by a uniform flow inclined by $45^{\circ}$ with respect to the background Cartesian grid and periodicity conditions at domain boundaries. The computational domain is a square $x, y \in[-15,+15]^{2}$ and the nondimensional velocity components are $u=v=1$. The vortex is initialized at $x_{0}=y_{0}=-10$ using the isentropic perturbations given by Yee et al. ${ }^{32}$ The flow is inviscid so that the exact solution is the passive advection of the vortex along the diagonal without dissipation. The background Cartesian mesh is composed of $100^{2}$ cells corresponding to approximately 4 cells across the vortex core radius. Space discretization is performed using the $3^{\text {rd }}$ - and $5^{t h}$-order FE-MUSCL schemes presented in Section II.B. For cases with VC, the VC term is of the same order as the baseline scheme and the confinement parameters are $\mu / \varepsilon=0.2, \varepsilon=0.16$ for FE-MUSCL3 and $\mu / \varepsilon=0.4, \varepsilon=0.02$ for FE-MUSCL5. Time discretization is performed using a classical 4-step Runge-Kutta scheme and a time step $\Delta t=0.025$.

Fig. 2 shows a comparison of tangential velocity profiles computed with FE-MUSCL schemes with and without VC. Curves represent cell-center values extracted horizontally from the position of minimum density. The effect of dissipative error is important for the $5^{t h}$-order baseline scheme and even more so for the $3^{\text {rd }}$ order one, leading to a complete diffusion of the vortex in a much shorter distance. VC achieves a significant reduction of this spurious dissipation, giving a solution that is considerably closer to the exact profile than those of baseline schemes, even though some spreading can be observed at the vortex boundary. This effect is reduced for the $5^{\text {th }}$-order solution compared to the $3^{\text {rd }}$-order one, but in any case the improvement with $\mathrm{VC}$ is significant for both cases, especially considering the coarse mesh resolution.
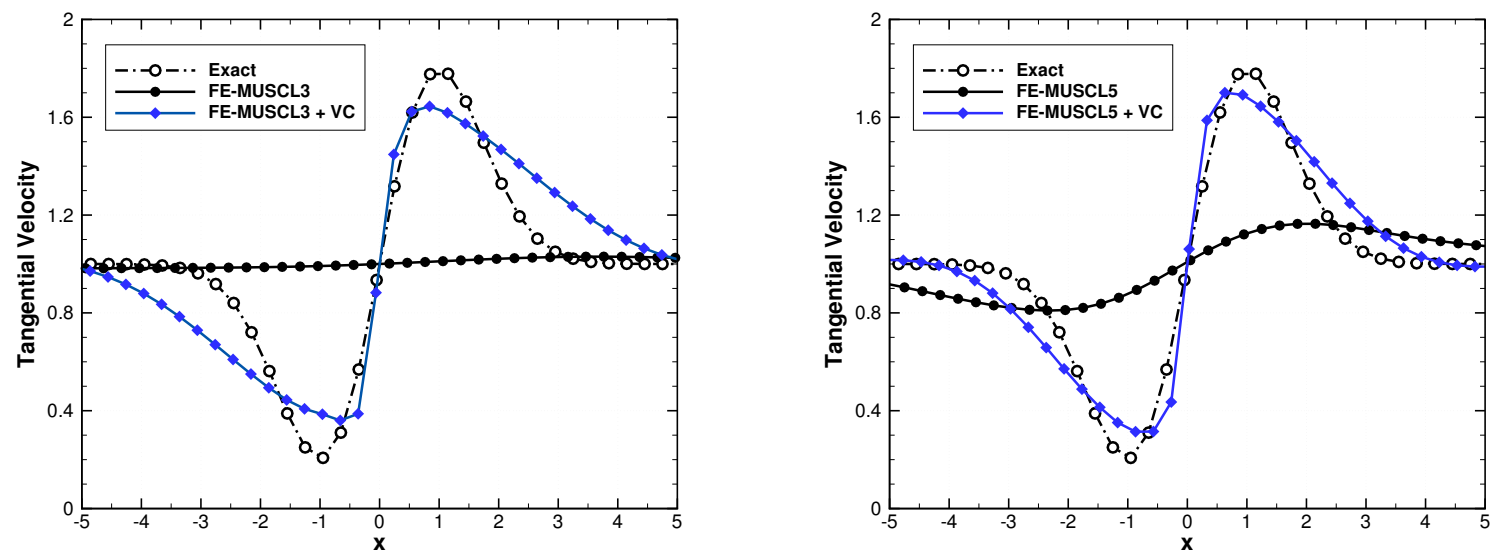

Figure 2. Comparison of tangential velocity profiles, centered at $x=0$, for FE-MUSCL schemes with and without VC. Profiles after 30 passages across the domain for the $3^{\text {rd }}$-order schemes (left) and after 300 passages across the domain for the $5^{\text {th }}$-order schemes (right).

\section{III.B. Viscous Taylor-Green Vortex}

The viscous Taylor-Green Vortex (TGV) ${ }^{33}$ is an excellent benchmark case for the evaluation of high-order methods, as it is characterized by kinetic energy transfer from larger to smaller scales, characteristic of a wide range of Large Eddy Simulation applications. Specifically the TGV case at $R e=1600$ has been used as a benchmark test case for recent high-order CFD workshops and high-precision numerical methods. ${ }^{34,35}$ Since it has been demonstrated that schemes with VC allow the advection of vortical structures over very long distances with low dissipation, the TGV case is chosen to evaluate these schemes in the calculation of multi-scale flow dynamics.

The TGV flow is an unsteady problem solved on a periodic computational domain $[2 \pi]^{3}$ with an analytical 
two-dimensional initial condition for velocity that corresponds to large-scale vortices:

$$
\begin{aligned}
u(x, y, z, 0) & =\sin x \cos y \cos z \\
v(x, y, z, 0) & =-\cos x \sin y \cos z \\
w(x, y, z, 0) & =0
\end{aligned}
$$

and an initial condition for pressure:

$$
p(x, y, z, 0)=p_{0}+\frac{\rho}{16}(\cos (2 x)+\cos (2 y))(\cos (2 z)+2)
$$

where $p_{0}=100$ and the Mach number is $M_{0}=0.10$. The initial density field is considered constant $\rho(x, y, z, 0)=\rho_{0}=1$. The fluid is considered a perfect gas with zero bulk viscosity, $\gamma=1.4$, and the Prandtl number is $\operatorname{Pr}=0.71$. All quantities have been adimensionalized with the reference velocity, density and length of the periodic box.

The compressible Navier-Stokes equations are solved on three different Cartesian meshes composed of 64, 128 and 256 cells in each mesh direction. Convective fluxes are discretized using a $5^{\text {th }}$-order accurate FE-MUSCL scheme, presented in Section II.B. Time integration is performed using an explicit 6-stage Runge-Kutta algorithm, formally accurate to $2^{n d}$-order, with optimized coefficients for minimal dispersive and dissipative error in the wavenumber space. ${ }^{36}$ The time step is set equal to $\Delta t=0.005, \Delta t=0.0025$ and $\Delta t=0.00125$ for the $64^{3}, 128^{3}$ and $256^{3}$ grids respectively so that the CFL number is approximately constant for the three cases. Previous studies with high-order VC alongside FE-MUSCL schemes have shown that good results can be obtained for $\varepsilon$ in the order of the baseline high-order artificial dissipation coefficient. The sensitivity to the choice of $\mu$ however, has not been assessed for complex flows. This study investigates three choices of the parameter $\mu / \varepsilon=\{0.0,0.2,0.4\}$ for $\varepsilon=1.2 k_{5}=0.02$. Results are compared against the reference computation of the International Workshop of High-Order CFD Methods, which is a converged DNS computation using a pseudo-spectral method on a $512^{3}$ mesh.

Fig. 3 shows the effect of $\mathrm{VC}$ on the rate of dissipation of kinetic energy. The integrated kinetic energy over the computational domain $\Omega$ at a specific time instant is:

$$
K=\frac{1}{\rho_{0} \Omega} \int_{\Omega} \frac{1}{2} \rho\|\vec{u}\|^{2} d \Omega
$$

and the dissipation rate is computed directly from the kinetic energy as $-d K / d t$. Vorticity Confinement is consistent with the complex dynamics of the TGV flow. The baseline scheme's dissipation rate is reduced during the initial stage, where the flow gradually generates smaller scales through the vortex stretching and tilting mechanisms characteristic of turbulence. This continues up to the dissipation peak where non-isotropic turbulence is fully developed and begins to decay due to the dissipation from the smallest scales. The peak value of the dissipation rate is largely dependent on the preservation of intermediate scales during the vortex stretching phase, and is therefore increased with the application of VC. Naturally, the peak value is reduced for increased values of $\mu / \varepsilon$ and the effect of $\mathrm{VC}$ diminishes with mesh refinement, in consistency with the reduced numerical error of the high-order flux discretization. The influence of the solution to the choice of $\mu / \varepsilon$ is small during the vortex stretching phase, especially for the finer $128^{3}$ and $256^{3}$ grids. Differences appear mostly at later times, as small variations in the preservation of vortices in the early decay phase (i.e. $t \approx 9-11$ ) lead to variations of their subsequent interaction.

A similar evaluation can be performed for the time evolution of integrated enstrophy over the computational domain:

$$
\mathrm{E}=\frac{1}{\rho_{0} \Omega} \int_{\Omega} \frac{1}{2} \rho\|\vec{\omega}\|^{2} d \Omega
$$

which for incompressible flow is related to the dissipation rate of kinetic energy as

$$
-\frac{d K}{d t}=2 \frac{\mu}{\rho_{0}} E
$$

where $\mu$ is the dynamic viscosity of the fluid. Vorticity gradients in Eq. (18) are calculated using the 7-point Dispersion-Relation-Preserving scheme of Tam \& Webb. ${ }^{37}$ Discrepancies from the relation in Eq. (19), between the time evolution of enstrophy and kinetic energy dissipation rate, are associated to errors due to compressibility and the dissipation of the numerical scheme. Specifically for the case of enstrophy, the large 


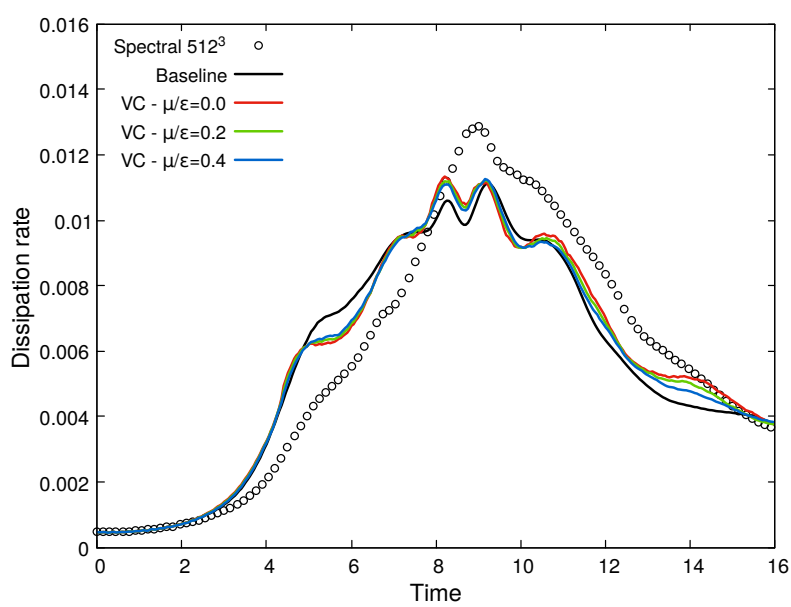

(a) $64^{3}$ mesh

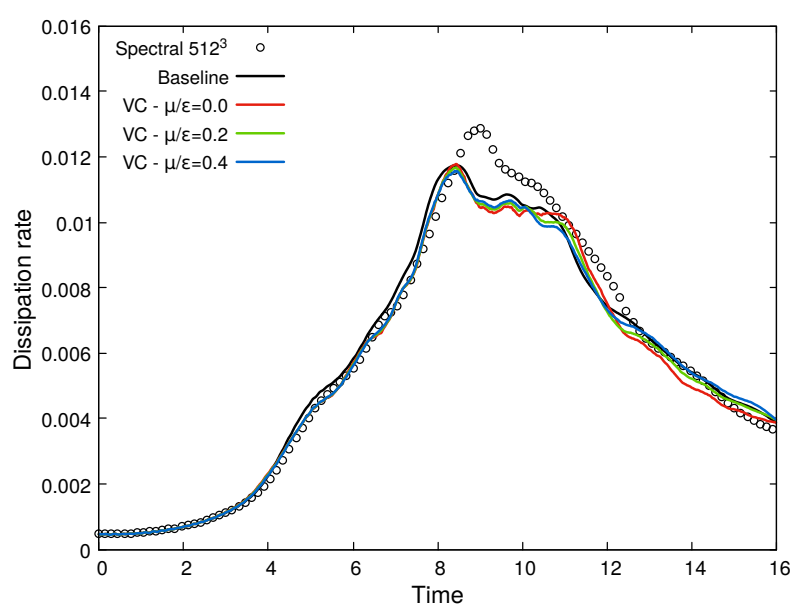

(b) $128^{3}$ mesh

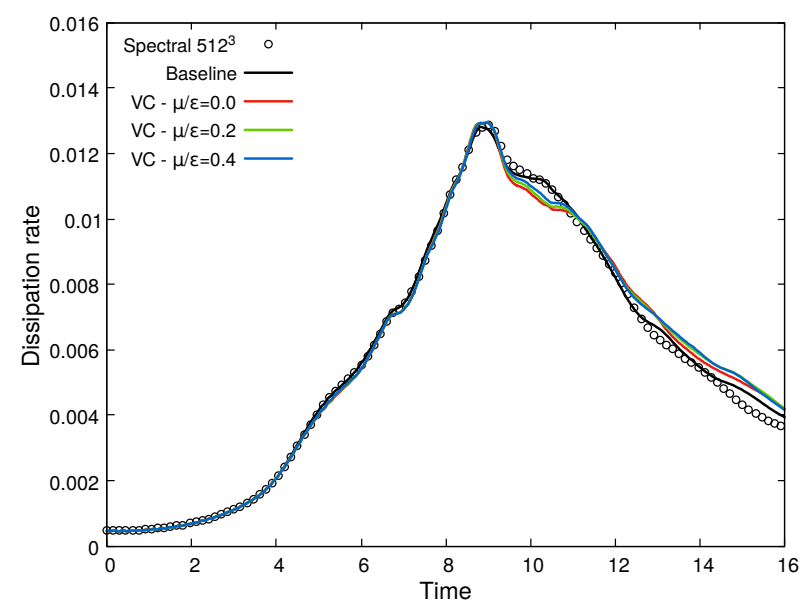

(c) $256^{3} \mathrm{mesh}$

Figure 3. Effect of $\mathrm{VC}$ on the evolution of the kinetic energy dissipation rate for the Taylor-Green vortex case and sensitivity to the value of $\mu / \varepsilon$. FE-MUSCL5 and $5^{t h}$-order Vorticity Confinement. 


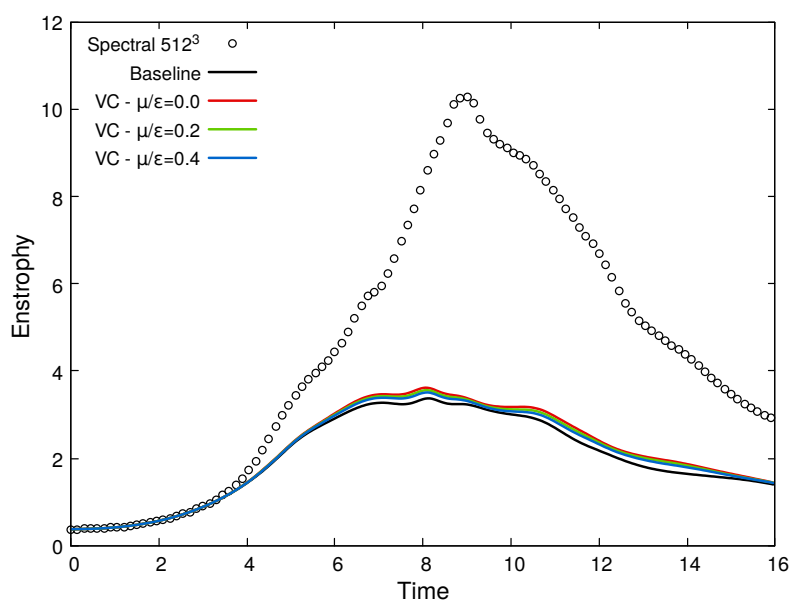

(a) $64^{3}$ mesh

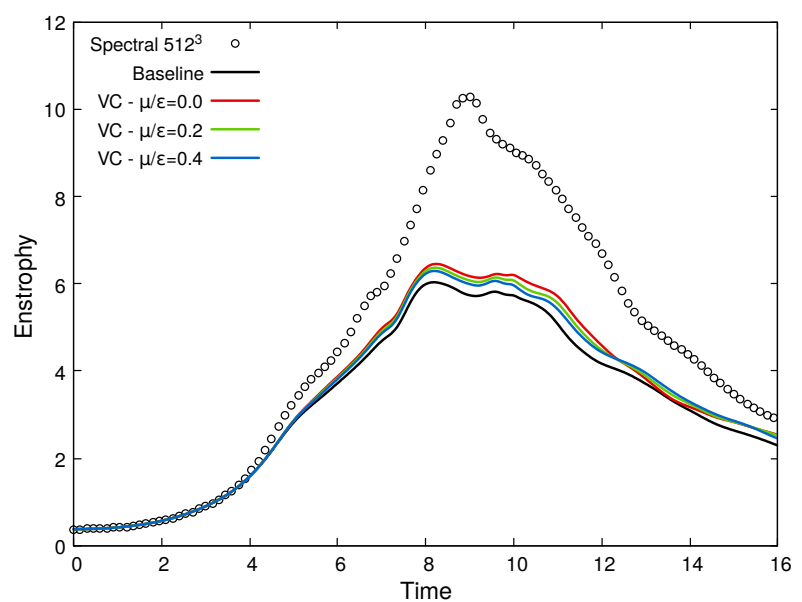

(b) $128^{3}$ mesh

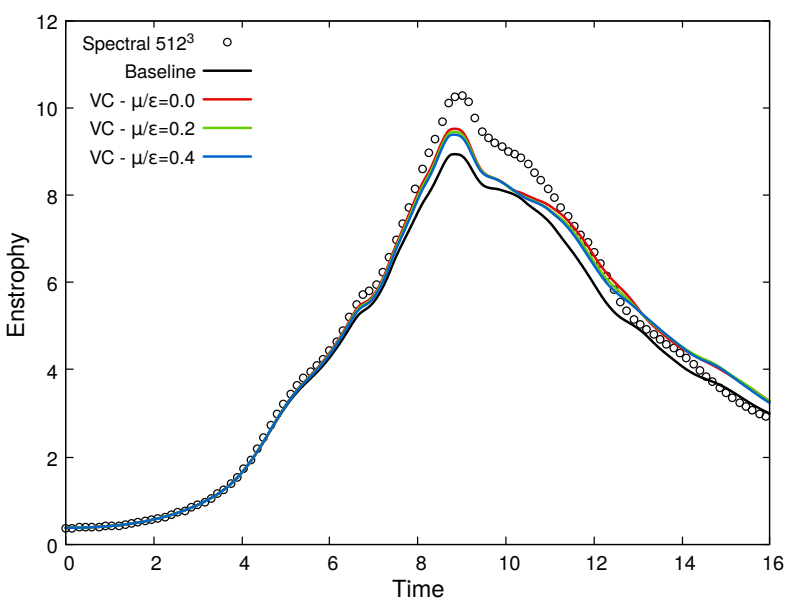

(c) $256^{3}$ mesh

Figure 4. Effect of VC on the evolution of enstrophy for the Taylor-Green vortex case and sensitivity to the value of $\mu / \varepsilon$. FE-MUSCL5 and $5^{\text {th }}$-order Vorticity Confinement. 
deviations from the reference solution are also a result of integrated error in the approximation of velocity gradients in Eq. (18), as opposed to Eq. (17) which includes the error in the approximation of conservative variables themselves. Results for the time evolution of enstrophy are shown in Fig. 4. As the high-order VC flux correction is explicitly based on vorticity, it naturally improves the approximation of enstrophy. It is important to note that the effect of $\mathrm{VC}$ does not diminish with mesh refinement, as was observed for the dissipation rate. This suggests that even though the magnitude of $\mathrm{VC}$ decreases with mesh refinement (according to the reduced numerical error of the baseline $5^{\text {th }}$-order scheme), the efficiency of $\mathrm{VC}$ in the calculation of velocity gradients is increased, possibly due to the improved alignment of the term with local vorticity. The sensitivity to the choice of $\mu / \varepsilon$ remains very small during the vortex stretching phase, but affects the value of the enstrophy peak. Last, the value of $\mu / \varepsilon$ seems to have an influence on the evolution of enstrophy in the late stages of turbulent decay. This influence is not observed on the $64^{3}$ grid due to the very coarse mesh size, but appears to be diminishing on the more refined $256^{3}$ case compared to the intermediate $128^{3}$.

\section{III.C. Decay of Compressible Homogeneous Isotropic Turbulence}

The next simulation concerns the decay of compressible Homogeneous Isotropic Turbulence (HIT). The initial turbulent flow field is divergence-free with no fluctuations of thermodynamic quantities. Fluctuations of velocity components are initialized following a prescribed Passot-Pouquet turbulent kinetic energy spectrum:

$$
E(\kappa)=A \kappa^{4} \exp \left(-2 \kappa^{2} / \kappa_{0}^{2}\right)
$$

where $A$ is a real coefficient that defines the initial volume averaged kinetic energy $K_{0}$ and $\kappa_{0}$ is the wavenumber corresponding to the energy spectrum peak. For this initial condition, the volume averaged kinetic energy $K_{0}$, volume averaged enstrophy $\Omega_{0}$, integral length scale $L_{I}$ and large-eddy-turnover time $\tau$ can be analytically derived from Eq. (20): ${ }^{38}$

$$
K_{0}=\frac{3 A}{64} \sqrt{2 \pi} \kappa_{0}{ }^{5} \quad \Omega_{0}=\frac{15 A}{256} \sqrt{2 \pi} \kappa_{0}{ }^{7} \quad L_{I}=\frac{\sqrt{2 \pi}}{\kappa_{0}} \quad \tau=\sqrt{\frac{32}{A}}(2 \pi)^{1 / 4} \kappa_{0}{ }^{-7 / 2}
$$

The fluid is considered as a perfect gas and the dependence of viscosity on temperature is assumed to follow a power-law $\mu=T^{0.76}$. Finally, the compressibility ratio in the initial condition was set to zero, as in Ref. ${ }^{39}$

Simulations were performed for Reynolds number $R e_{\lambda}=175$ based on the Taylor microscale, and the energy spectrum peak was chosen at $\kappa_{0}=4$. Two conditions for the initial turbulent Mach number were investigated, $M_{t 0}=0.2$ and $M_{t 0}=0.5$, up to which the influence of thermodynamic fluctuations has been found to be small $^{38}$ and compressibility effects are moderate, allowing calculation using the FE-MUSCL schemes presented in Section II.B. Higher initial turbulent Mach number cases require the use of shockcapturing schemes such as pure Jameson-like or mixed Jameson-Ducros high-order artificial dissipation, as in Ref. ${ }^{40}$ High-order VC can also be applied in this case as it is formulated independently of the baseline scheme. It is expected to introduce negligible modifications of the scheme's shock-capturing properties, as dissipation near shocks (including the high-order negative dissipation of $\mathrm{VC}$ ) is dominated by the low-order dissipation term. For low turbulent Mach number cases however, the FE-MUSCL schemes of Section II.B, which use a matrix-form of dissipation, yield more precise results.

The case is solved on a periodic computational domain $[2 \pi]^{3}$, discretized by a Cartesian grid composed of $128^{3}$ cells. This corresponds to Implicit Large-Eddy Simulations (ILES), since the grid does not suffice for the adequate resolution of the Kolmogorov microscales, and the role of small-scale turbulent dissipation is taken up by the scheme's high-order numerical dissipation, rather than by an explicit subgrid scale model. Computations are performed up to time $t=10 \tau$ and time integration is performed by an explicit 6 -stage Runge-Kutta algorithm with optimized coefficients, ${ }^{36}$ as in Section III.B. Cases with VC use the $5^{\text {th }}$-order accurate FE-MUSCL scheme of Section II.B and different values of confinement parameters, summed up in Table 2. For these cases, the effect of $\mathrm{VC}$ is evaluated in comparison with the baseline scheme without confinement and a reference solution computed with a $9^{\text {th }}$-order accurate upwind FE-MUSCL scheme on the same grid.

The HIT evolution consists of a vortex stretching phase, where viscous effects are negligible and enstrophy increases up to a peak value, and a subsequent decay phase characterized by viscous dissipation at the Kolmogorov microscales. The resolvability of the enstrophy peak is often used as measure of the resolution of numerical schemes. Fig. 5 shows the evolution of enstrophy obtained with FE-MUSCL schemes with 
Table 2. Numerical parameters for the different cases with VC

\begin{tabular}{lcccc}
\hline \hline & Baseline scheme & VC order & $\varepsilon$ & $\mu / \varepsilon$ \\
\hline Case 1 & FE-MUSCL5 & $5^{t h}$ & $1.2 k_{5}$ & 0.00 \\
Case 2 & FE-MUSCL5 & $5^{t h}$ & $1.2 k_{5}$ & 0.20 \\
Case 3 & FE-MUSCL5 & $5^{t h}$ & $2.4 k_{5}$ & 0.00 \\
\hline \hline
\end{tabular}

and without VC for the two different initial turbulent Mach number cases. Schemes with VC manage to predict an increased enstrophy peak compared to the baseline $5^{\text {th }}$-order FE-MUSCL scheme for all cases. Furthermore, the instant where the enstrophy peak occurs is slightly delayed for schemes with less numerical dissipation. Naturally, the effect of $\mathrm{VC}$ is mainly driven by the value of $\varepsilon$ as this parameter defines the magnitude of the $\mathrm{VC}$ term. Good results are obtained for $\varepsilon$ close to the value of the $5^{\text {th }}$-order artificial dissipation coefficient, in consistency with previous studies. An augmented value of this parameter gives a further increased dissipation peak, which at $M_{t 0}=0.5$ is comparable to the reference $9^{\text {th }}$-order solution, but results in slightly higher enstrophy values compared to the reference during the late time instants (Case 3 ).

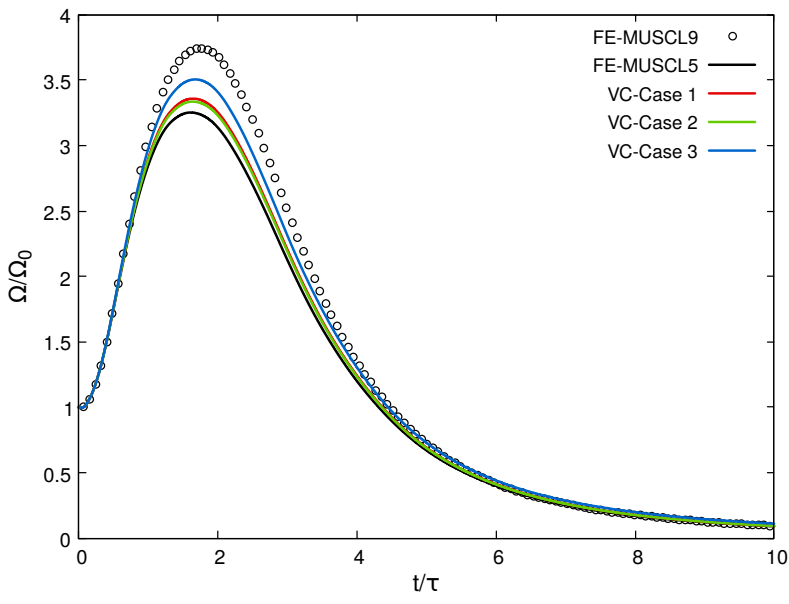

(a) $M_{t 0}=0.2$

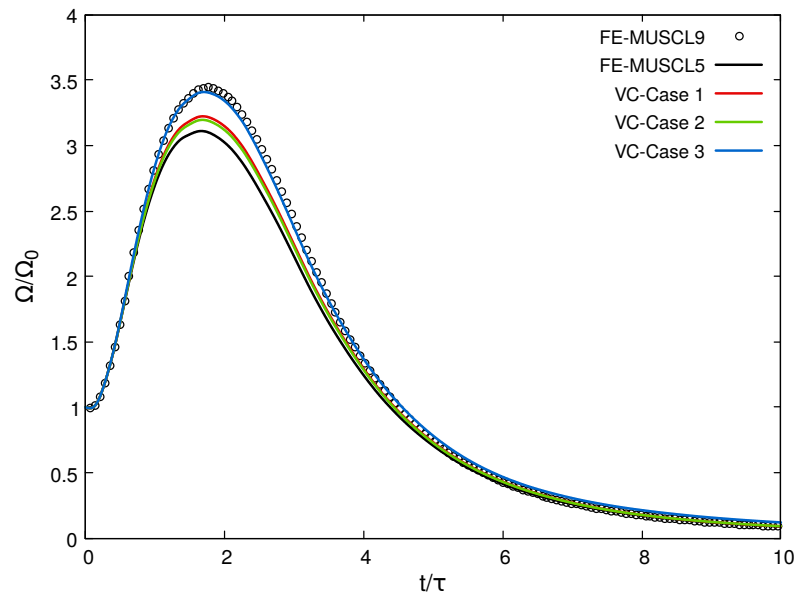

(b) $M_{t 0}=0.5$

Figure 5. Comparison of the time evolution of enstrophy obtained with FE-MUSCL schemes with and without VC for the two different initial turbulent Mach number cases.

The influence of $\mathrm{VC}$ on the evolution of thermodynamic quantities is monitored through the time evolution of the root-mean-square (r.m.s.) of pressure fluctuations, presented in Figs. 6-7. These results show little sensitivity to the space discretization scheme, with differences appearing mainly close to the time of the enstrophy peak (before $t=2 \tau$ ). The effect of $\mathrm{VC}$ on the evolution of $p_{r m s}$ is found to be small for all cases. Close-up views show that results with VC are very close to the reference $9^{t h}$-order computation, at least for Cases 1-2. The influence of the choice of $\mu / \varepsilon$ between the two latter Cases is found to be small, the results being superimposed within plotting accuracy. Similar trends are observed for the time evolution of the r.m.s. values of density and temperature fluctuations.

An interesting comparison can be made by considering the time evolution of high-order statistical moments of the turbulent flow. Comparisons are presented for the skewness and flatness factor:

$$
\mathrm{S}(\bullet) \triangleq \frac{\left\langle(\bullet)^{3}\right\rangle}{\left\langle(\bullet)^{2}\right\rangle^{3 / 2}} \quad \mathrm{~F}(\bullet) \triangleq \frac{\left\langle(\bullet)^{4}\right\rangle}{\left\langle(\bullet)^{2}\right\rangle^{2}}
$$

of the fluctuating velocity gradient distribution, where $\langle\bullet\rangle$ denotes a volume average over the computational domain at a fixed time instant. These quantities represent measures of the difference of the velocity fluctuation field from a Gaussian distribution and are generally more sensitive to the space discretization scheme. Typical values of the skewness factor are $\mathrm{S}=-0.4 \pm 0.1$ for incompressible HIT. ${ }^{41}$ The time evolution of the skewness factor is presented in Fig. 8 for the two turbulent Mach number cases. Cases with VC do not provide results closer to the reference than the baseline $5^{t h}$-order scheme. Specifically, the skewness factor for 

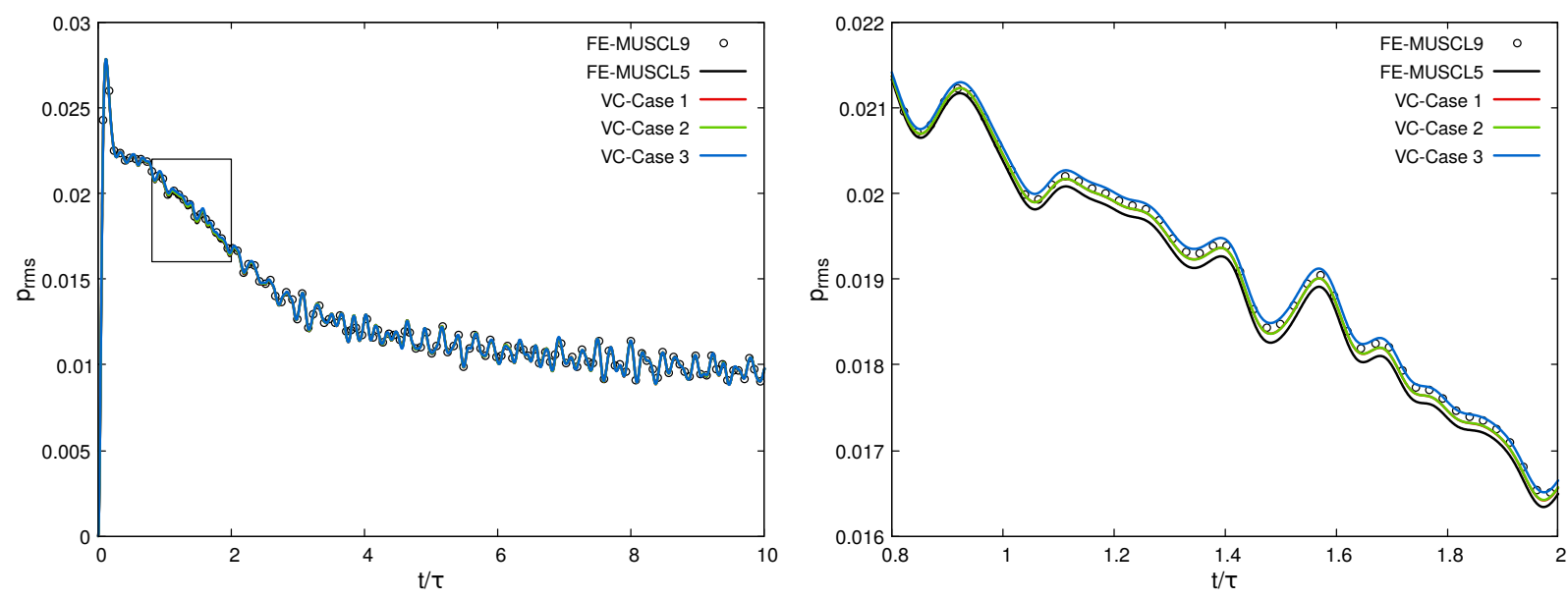

Figure 6. Comparison of the time evolution of r.m.s. pressure obtained with FE-MUSCL schemes with and without VC for initial turbulent Mach number $M_{t 0}=0.2$. Evolution during the complete computed time (left) and detail at $t=0.8 \tau-2 \tau$ (right).
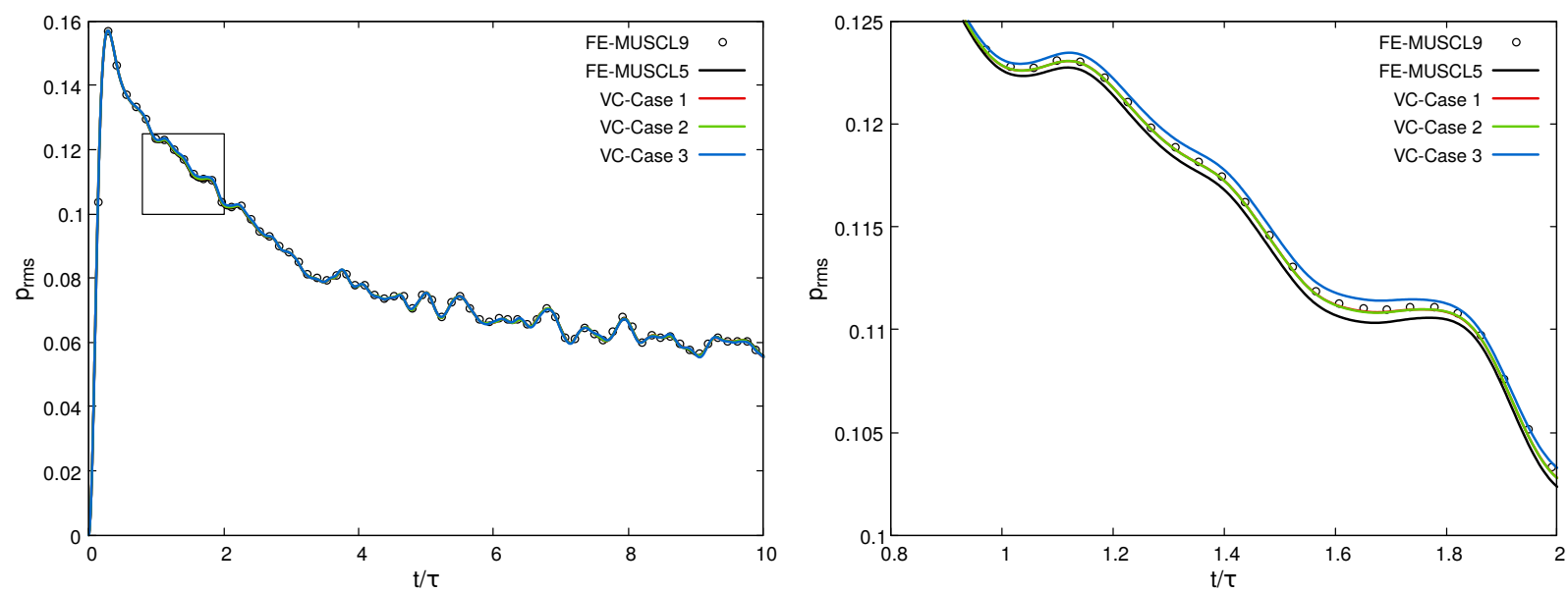

Figure 7. Comparison of the time evolution of r.m.s. pressure obtained with FE-MUSCL schemes with and without VC for initial turbulent Mach number $M_{t 0}=0.5$. Evolution during the complete computed time (left) and detail at $t=0.8 \tau-2 \tau$ (right) 
Case 3 seems to diverge over long times, especially for the $M_{t 0}=0.5$ case. Cases 1-2 however give consistent results, very close to those of the baseline schemes.

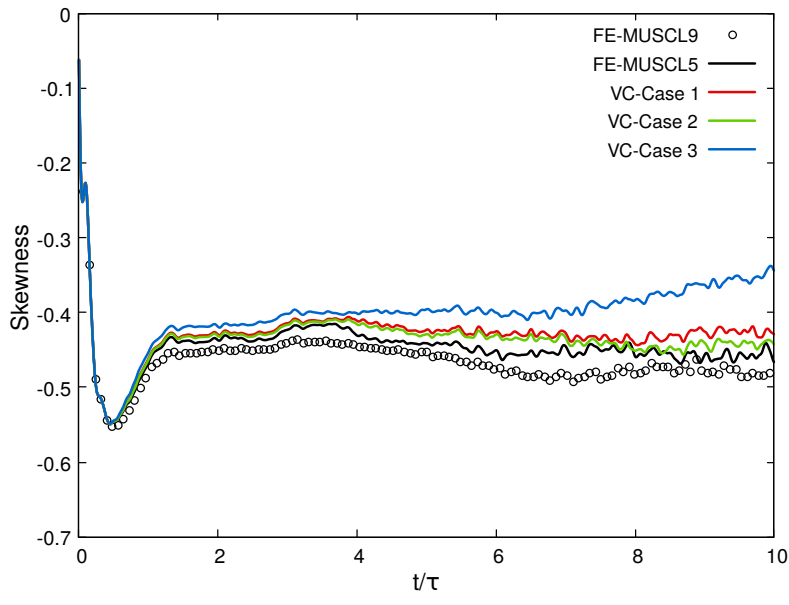

(a) $M_{t 0}=0.2$

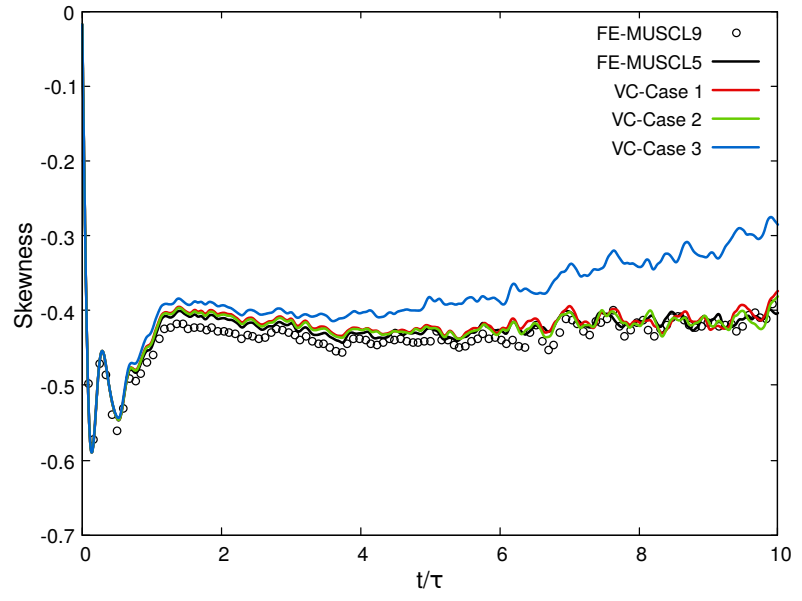

(b) $M_{t 0}=0.5$

Figure 8. Comparison of the time evolution of the skewness factor of the fluctuating velocity gradient distribution obtained with FE-MUSCL schemes with and without VC for the two different initial turbulent Mach number cases.

The time evolution of the flatness factor is presented in Fig. 9. Schemes with VC give consistent results with smaller differences from the baseline than for the time evolution of the skewness factor (Fig. 8). An exception is again observed for Case 3, which displays a larger difference from the baseline than Cases 1-2, and follows a different trend at late times for the $M_{t 0}=0.5$ case.

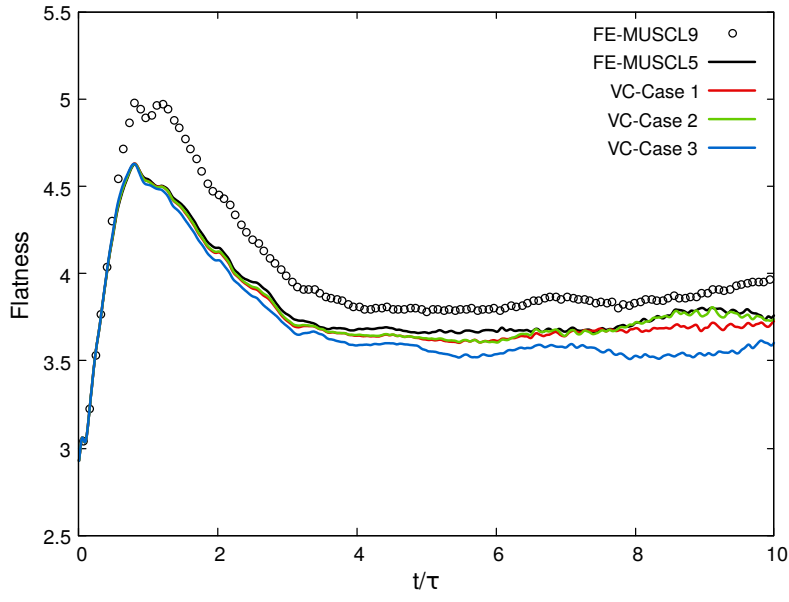

(a) $M_{t 0}=0.2$

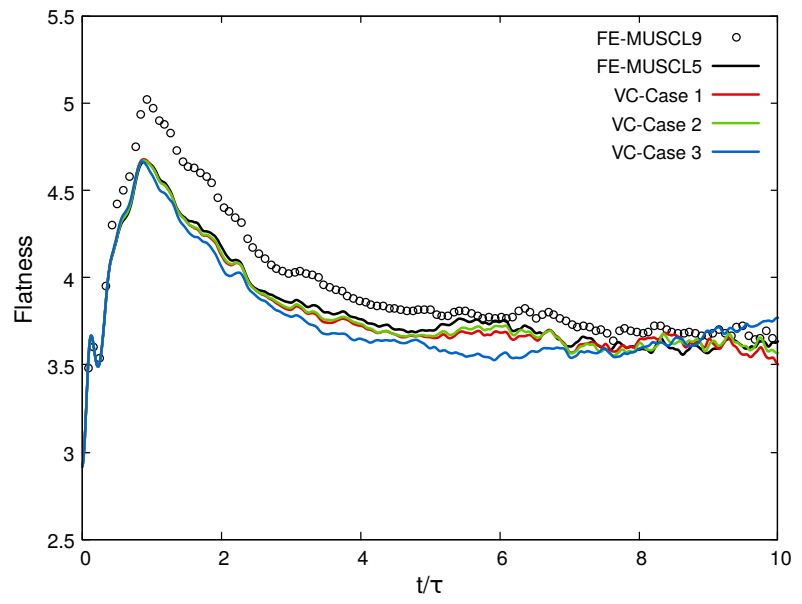

(b) $M_{t 0}=0.5$

Figure 9. Comparison of the time evolution of the flatness factor of the fluctuating velocity gradient distribution obtained with FE-MUSCL schemes with and without VC for the two different initial turbulent Mach number cases.

The resolvability of numerical schemes is further depicted on the turbulent kinetic energy spectrum, presented in Fig. 10 for different time instants during the HIT decay. An inertial range following the $-5 / 3$ law has not been fully developed for the low Reynolds number $R e_{\lambda}$ of this study, but differences in the energy spectrum are observed due to the schemes' numerical dissipation, even at times as early as $t=2 \tau$. The application of VC extends the cut-off wavenumber of the baseline $5^{\text {th }}$-order FE-MUSCL scheme for all cases, the differences being naturally more important at later times, due to the accumulation of numerical dissipation for the baseline scheme. As discussed previously, high-values of the confinement parameter $\varepsilon$ lead to the negative dissipation of $\mathrm{VC}$ being very large compared to the dissipation of the baseline scheme, thus causing reduced dissipation and accumulation of energy at high wavenumbers during the late stages of the decay (Case 3). This is possibly related to the increased enstrophy at late times, observed previously in Fig. 

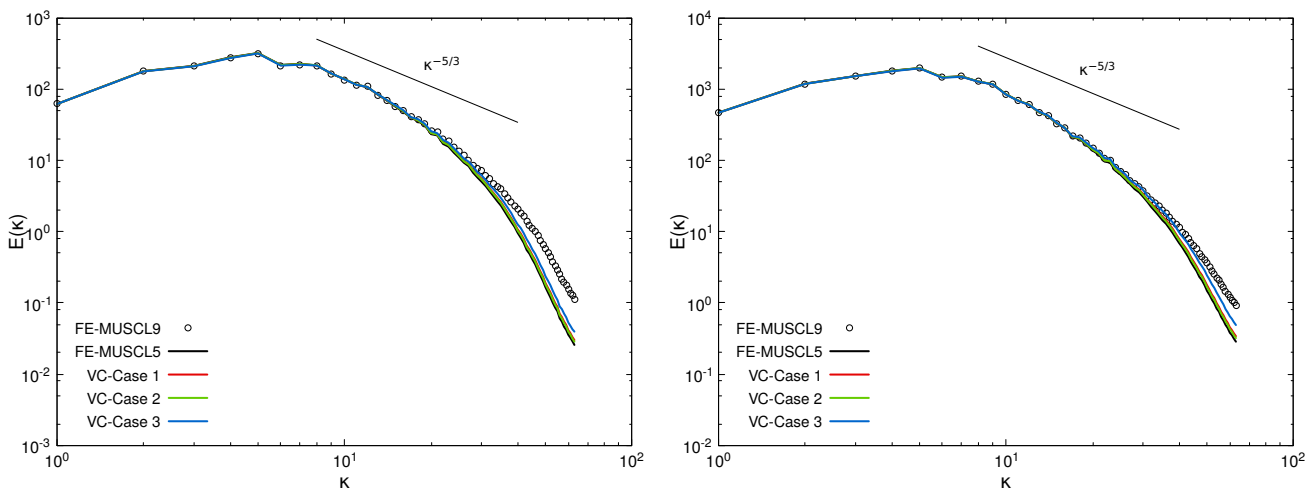

(a) $t=2 \tau$
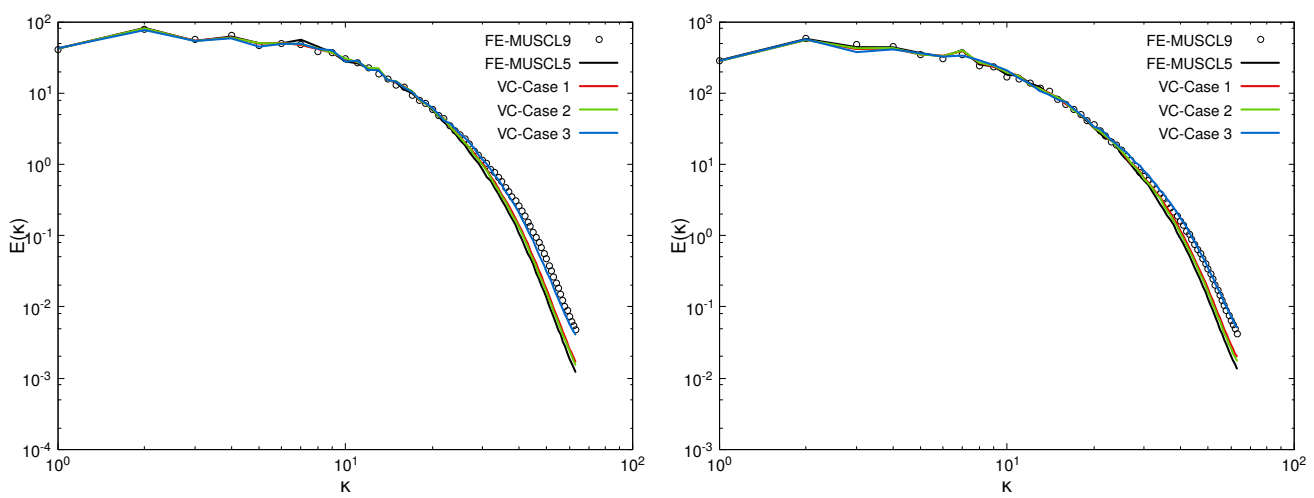

(b) $t=5 \tau$
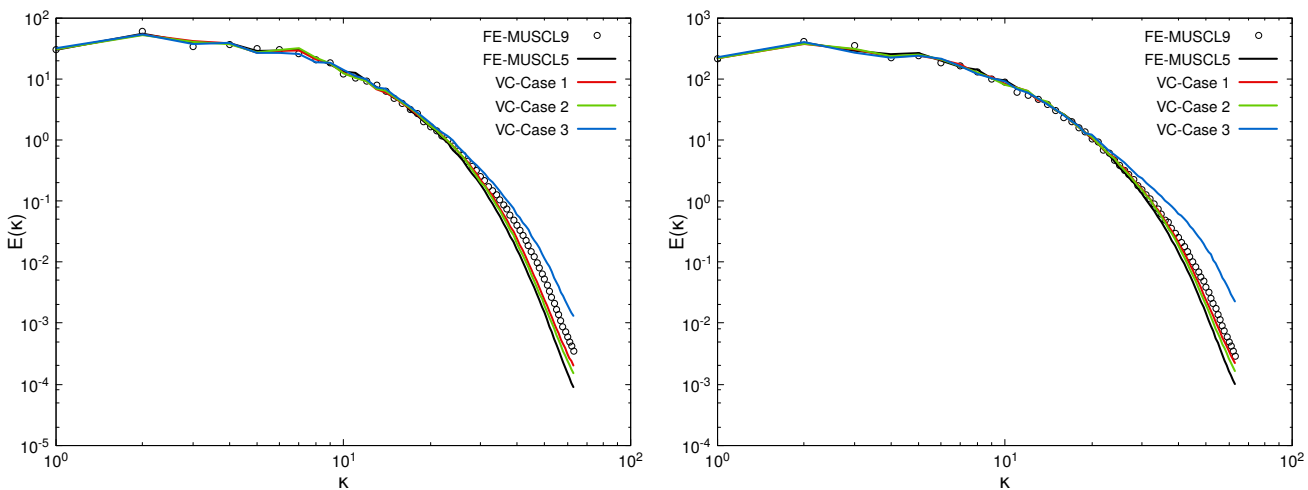

(c) $t=7 \tau$
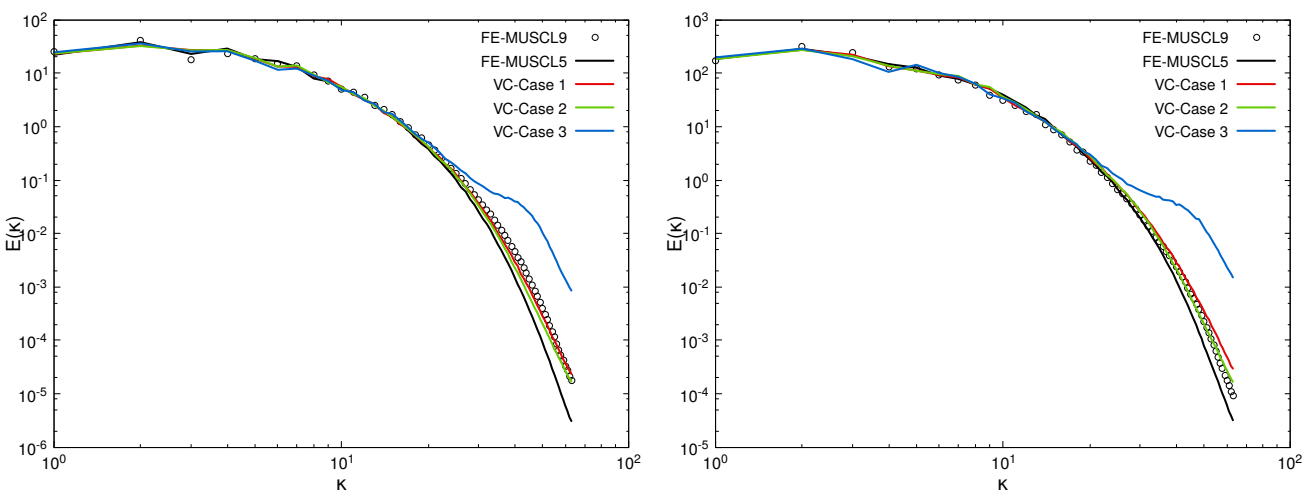

(d) $t=10 \tau$

Figure 10. Comparison of the turbulent kinetic energy spectrum at different time instants during the HIT decay obtained with FE-MUSCL schemes with and without VC for initial turbulent Mach number $M_{t 0}=0.2$ (left) and $M_{t 0}=0.5$ (right).

\section{5 of 18}


5 for the same Case. Such values are possibly more suitable for an explicit Large-Eddy Simulation approach, so that the dissipation of the baseline scheme is efficiently balanced by $\mathrm{VC}$ and small-scale dissipation is introduced in the form of an explicit subgrid scale (SGS) model. Reasonable and consistent results are however obtained for $\varepsilon$ closer to the value of the high-order artificial dissipation coefficient (Cases 1-2).

Fig. 11 shows iso-surfaces of constant vorticity magnitude at $t=10 \tau$, colored by the local Mach number. Results are in agreement with previous observations. Specifically, high values of the parameter $\varepsilon$ (Case 3 ) lead to overly reduced dissipation at smaller scales and accumulation of small structures in the domain at late times. However for values of $\varepsilon$ close to the high-order artificial dissipation coefficient, the solution is consistent with the reference and the preservation of turbulent structures is noticeably improved compared to the baseline $5^{\text {th }}$-order FE-MUSCL scheme (Case 1).

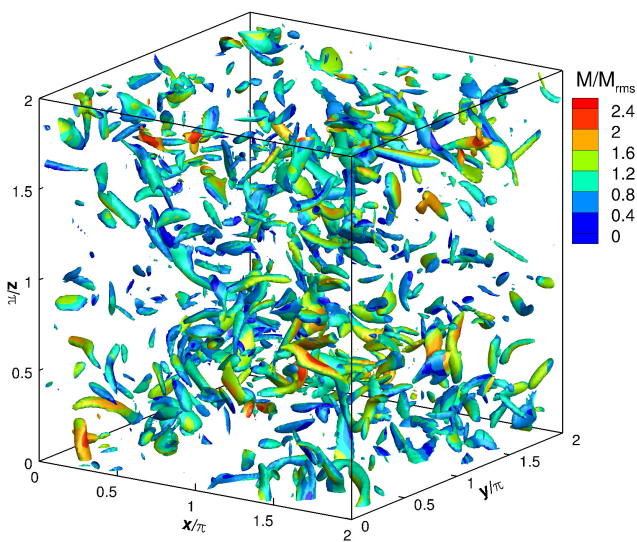

(a) FE-MUSCL9

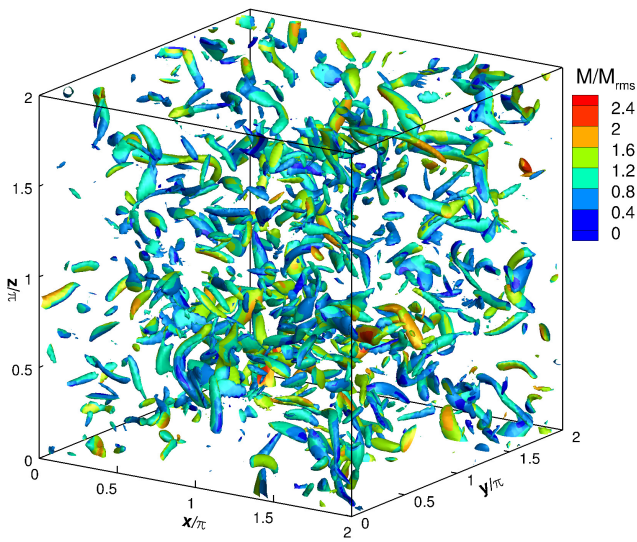

(c) VC Case 1

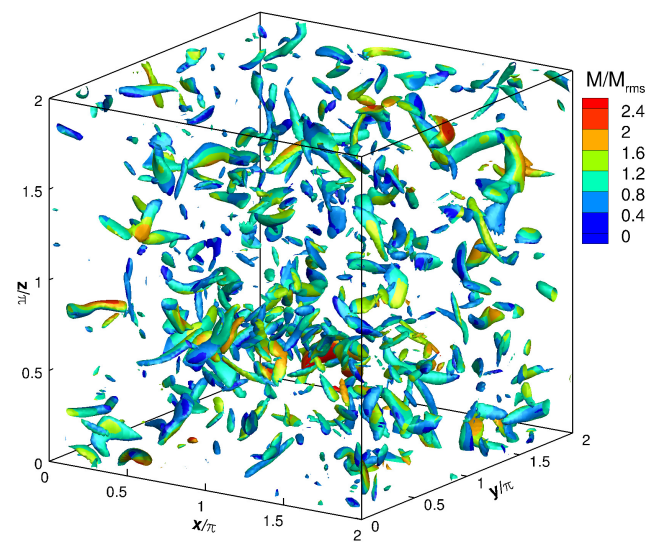

(b) FE-MUSCL5

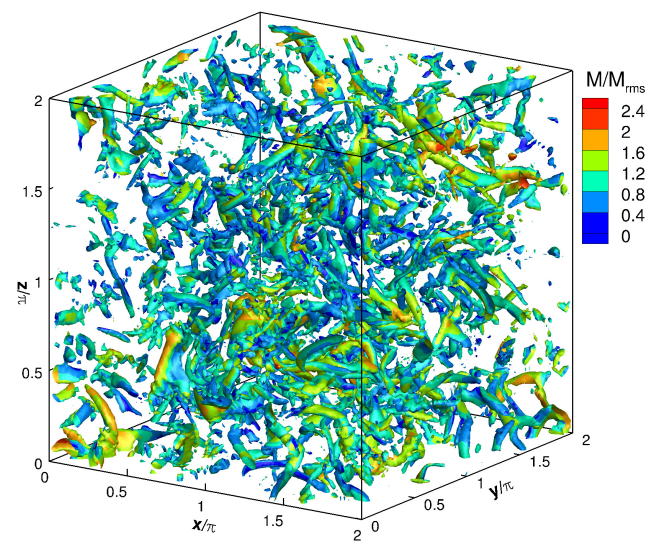

(d) VC Case 3

Figure 11. Iso-surfaces of constant vorticity magnitude at $t=10 \tau$ colored by the local Mach number. Computed with FE-MUSCL schemes with and without VC on a $128^{3}$ grid for initial turbulent Mach number $M_{t 0}=0.2$.

\section{Conclusions}

This paper presents results from the application of high-order Vorticity Confinement schemes to compressible vortical flows. The cases are primarily aimed at evaluating the consistency of these schemes with complex vortical dynamics and their applicability to turbulent flow simulations. In vortex advection studies, high-order VC schemes were shown to effectively balance the baseline numerical dissipation and to be capable of propagating structures over long distances on coarse grids with low diffusion. For the more complex Taylor-Green vortex and homogeneous isotropic turbulence decay cases, high-order VC schemes were shown to accommodate the complex turbulent interactions without requiring special treatment. Constant values 
of the confinement parameters $\mu$ and $\varepsilon$ in space and time were found to be adequate and the sensitivity to their choice was investigated. Specifically, values of $\varepsilon$ close to the high-order artificial dissipation coefficient were shown to work well, in consistency with previous applications of these schemes, whereas high values appear to overly reduce small-scale dissipation and lead to the accumulation of energy at the smallest scales. The sensitivity to the choice of $\mu$ was found to be moderate for reasonable values of the ratio $\mu / \varepsilon$ and even negligible during the treatment of large-scale structures. Furthermore, it was found that the artificial dissipation of the VC term (i.e. the coefficient $\mu$ ) can be set to zero without affecting the stability of the scheme in turbulent flow calculations, at least alongside the upwind dissipative FE-MUSCL baseline schemes used in the present work. Last, for the cases investigated in this paper, the effect of VC was found to be more important in the evolution of enstrophy or the turbulent kinetic energy spectrum, and less on the evolution of thermodynamic quantities.

\section{Acknowledgments}

This research was co-funded by ONERA and a DGA-MRIS scholarship under the convention 2014-600055 .

\section{References}

\footnotetext{
${ }^{1}$ Bauknecht, A., Ewers, B., Wolf, C., Leopold, F., Yin, J., and Raffel, M., "Three-dimensional reconstruction of helicopter blade-tip vortices using a multi-camera BOS system," Experiments in Fluids, Vol. 56, No. 1, 2015, pp. 1866.

${ }^{2}$ Desse, J. and Olchewsky, F., "Digital Holographic Interferometry for Analysing HighDensity Gradients in Fluid Mechanics," Holographic Materials and Optical Systems, edited by I. Naydenova, D. Nazarova, and T. Babeva, chap. 13, InTech, Rijeka, 2017.

${ }^{3}$ Cottet, G.-H. and Koumoutsakos, P. D., Vortex methods: theory and practice, Cambridge University Press, 2000.

${ }^{4}$ Winckelmans, G., Cocle, R., Dufresne, L., and Capart, R., "Vortex methods and their application to trailing wake vortex simulations," Comptes Rendus Physique, Vol. 6, No. 45, 2005, pp. 467 - 486, Aircraft trailing vortices.

${ }^{5}$ Govindarajan, B. and Leishman, J., "Curvature Corrections to Improve the Accuracy of Free-Vortex Methods," Journal of Aircraft, Vol. 53, No. 2, 2015, pp. 378-386.

${ }^{6}$ de la Llave Plata, M., Couaillier, V., Marmignon, C., Le Pape, M.-C., Gazaix, M., and Cantaloube, B., "Further developments in the multiblock hybrid CFD solver elsA-H," 50th AIAA Aerospace Sciences Meeting, AIAA paper 2012-1112, 2012 .

${ }^{7}$ Sankaran, V., Wissink, A., Datta, A., Sitaraman, J., Potsdam, M., Jayaraman, B., Katz, A., Kamkar, S., Roget, B., Mavriplis, D., Saberi, H., Chen, W.-B., Johnson, W., and Strawn, R., "Overview of the Helios Version 2.0 Computational Platform for Rotorcraft Simulations," Aerospace Sciences Meetings, American Institute of Aeronautics and Astronautics, Jan. 2011, pp. -.

${ }^{8}$ Papadakis, G. and Voutsinas, S. G., "In view of accelerating CFD simulations through coupling with vortex particle approximations," Journal of Physics: Conference Series, Vol. 524, IOP Publishing, 2014, p. 012126.

${ }^{9}$ Kamkar, S., Wissink, A. M., Sankaran, V., and Jameson, A., "Feature-driven Cartesian adaptive mesh refinement for vortex-dominated flows," Journal of Computational Physics, Vol. 230, No. 16, 2011, pp. 6271-6298.

${ }^{10}$ Fidkowski, K. and Roe, P., "An entropy adjoint approach to mesh refinement," SIAM Journal on Scientific Computing, Vol. 32, No. 3, 2010, pp. 1261-1287.

${ }^{11}$ Haider, F., Discretization on general unstructured grids and applications on LES, Ph.D. thesis, Université Pierre et Marie Curie - Paris VI, 2009.

${ }^{12}$ Speziale, C. G., "On the advantages of the vorticity-velocity formulation of the equations of fluid dynamics," Journal of Computational Physics, Vol. 73, No. 2, 1987, pp. $476-480$.

${ }^{13}$ Davies, C. and Carpenter, P. W., "A Novel Velocity Vorticity Formulation of the NavierStokes Equations with Applications to Boundary Layer Disturbance Evolution," Journal of Computational Physics, Vol. 172, No. 1, 2001, pp. 119 - 165.

${ }^{14}$ Morton, K. and Roe, P. L., "Vorticity-Preserving Lax-Wendroff-Type Schemes for the System Wave Equation," SIAM Journal on Scientific Computing, Vol. 23, No. 1, 2001, pp. 170-192.

${ }^{15}$ Falissard, F., Lerat, A., and Sidès, J., "Computation of airfoil-vortex interaction using a vorticity-preserving scheme," AIAA Journal, Vol. 46, No. 7, 2008, pp. 1614-1623.

${ }^{16}$ Steinhoff, J. and Lynn, N., Treatment of Vortical Flow Using Vorticity Confinement, Frontiers of Computational Fluid Dynamics, World Scientific, 2006, pp. 199-237.

${ }^{17}$ Steinhoff, J., Lynn, N., and Wang, L., Large eddy simulation using vorticity confinement, Chapter 4 of Implicit Large Eddy Simulations: Computing Turbulent Flow Dynamics, Cambridge University Press, 2006.

${ }^{18}$ Steinhoff, J., Lynn, N., Yonghu, W., Fan, M., Wang, L., and Dietz, W., Turbulent flow simulations using vorticity confinement, Chapter 12 of Implicit Large Eddy Simulations: Computing Turbulent Flow Dynamics, Cambridge University Press, 2006.

${ }^{19}$ Costes, M., Petropoulos, I., and Cinnella, P., "Development of a third-order accurate vorticity confinement scheme," Computers \& Fluids, Vol. 136, 2016, pp. 132-151.
} 
${ }^{20}$ Costes, M. and Juillet, F., "Analysis and higher-order extension of the VC2 confinement scheme," Computers E Fluids, Vol. 56, No. 0, 2012, pp. $102-117$.

${ }^{21}$ Petropoulos, I., Costes, M., and Cinnella, P., "Development and analysis of high-order vorticity confinement schemes," Computers \& Fluids, 2017, pp. -, DOI: http://doi.org/10.1016/j.compfluid.2017.04.011.

${ }^{22}$ Steinhoff, J. and Underhill, D., "Modification of the Euler equations for vorticity confinement: Application to the computation of interacting vortex rings," Physics of Fluids, Vol. 6, No. 8, 1994, pp. 2738-2744.

${ }^{23}$ Lynn, N., Investigation of Vorticity Confinement as a high-Reynolds number turbulence model, Ph.D. thesis, University of Tennessee Space Institute, 2007.

${ }^{24}$ Hirsch, C., Numerical Computation of Internal and External Flows: The Fundamentals of Computational Fluid Dynamics, Butterworth-Heinemann, 2007.

${ }^{25}$ Lerat, A. and Corre, C., High-order residual-based compact schemes on structured grids, in CFD-Higher order discretization methods, VKI-LS 2006-01, von Karman Institute for Fluid Dynamics, November 2006.

${ }^{26}$ Steinhoff, J., Puskas, E., Babu, S., Wenren, Y., and Underhill, D., "Computation of thin features over long distances using solitary waves," AIAA paper 97-1976, 13th Computational Fluid Dynamics Conference, 13th Computational Fluid Dynamics Conference, Snowmass, Colorado, June 1997.

${ }^{27}$ Steinhoff, J., Dietz, W., Haas, S., Xiao, M., Lynn, N., and Fan, M., "Simulating small scale features in fluid dynamics and acoustics as nonlinear solitary waves," AIAA paper 2003-078, 41st Aerospace Meeting and Exhibit, Reno, Nevada, January 2003.

${ }^{28}$ Haller, G., "An objective definition of a vortex," Journal of Fluid Mechanics, Vol. 525, 2005, pp. 1-26.

${ }^{29}$ Outtier, P.-Y., Content, C., Cinnella, P., and Michel, B., "The high-order dynamic computational laboratory for CFD research and applications," Fluid Dynamics and Co-located Conferences, American Institute of Aeronautics and Astronautics, June 2013, pp. -.

${ }^{30}$ Costes, M., "Stability analysis of the VC2 confinement scheme for the linear transport equation," Computers 83 Fluids, Vol. 86, No. 0, 2013, pp. $537-557$.

${ }^{31}$ Pirozzoli, S., "On the spectral properties of shock-capturing schemes," Journal of Computational Physics, Vol. 219, No. 2, 2006, pp. $489-497$.

${ }^{32}$ Yee, H., Vinokur, M., and Djomehri, M., "Entropy Splitting and Numerical Dissipation," Journal of Computational Physics, Vol. 162, No. 1, 2000, pp. $33-81$.

${ }^{33}$ Taylor, G. I. and Green, A. E., "Mechanism of the Production of Small Eddies from Large Ones," Proceedings of the Royal Society of London A: Mathematical, Physical and Engineering Sciences, Vol. 158, No. 895, 1937, pp. $499-521$.

${ }^{34}$ Bull, J. R. and Jameson, A., "Simulation of the Taylor-Green vortex using high-order flux reconstruction schemes," AIAA Journal, Vol. 53, No. 9, 2015, pp. 2750-2761.

${ }^{35}$ Van Rees, W. M., Leonard, A., Pullin, D., and Koumoutsakos, P., "A comparison of vortex and pseudo-spectral methods for the simulation of periodic vortical flows at high Reynolds numbers," Journal of Computational Physics, Vol. 230, No. 8, 2011, pp. 2794-2805.

${ }^{36}$ Bogey, C. and Bailly, C., "A family of low dispersive and low dissipative explicit schemes for flow and noise computations," Journal of Computational Physics, Vol. 194, No. 1, 2004, pp. 194-214.

${ }^{37}$ Tam, C. K. W. and Webb, J. C., "Dispersion-relation-preserving finite difference schemes for computational acoustics," Journal of Computational Physics, Vol. 107, No. 2, 1993, pp. 262-281.

${ }^{38}$ Samtaney, R., Pullin, D. I., and Kosović, B., "Direct numerical simulation of decaying compressible turbulence and shocklet statistics," Physics of Fluids, Vol. 13, No. 5, 2001, pp. 1415-1430.

${ }^{39}$ Sciacovelli, L., Cinnella, P., and Grasso, F., "Dense gas effects in inviscid homogeneous isotropic turbulence," Journal of Fluid Mechanics, Vol. 800, 2016, pp. 140-179.

${ }^{40}$ Cinnella, P. and Content, C., "High-order implicit residual smoothing time scheme for direct and large eddy simulations of compressible flows," Journal of Computational Physics, Vol. 326, 2016, pp. $1-29$.

${ }^{41}$ Sagaut, P. and Cambon, C., Homogeneous Turbulence Dynamics, Cambridge University Press, 2008. 